\title{
COMPARISON OF TWO PERIPHERAL BLOCK METHODS FOR POSITION PAIN IN FEMORAL NECK FRACTURES
}

\author{
Unal H. *, Baskan S. ${ }^{*}$, Aytac I. ${ }^{*}$, Alkan M. ${ }^{*}$,Baydar ${ }^{*}$ M. \\ ${ }^{*}$ Ankara Numune Training and Research Hospital
}

BACKGROUND: In cases of femoral neck fractures, spinal, or epidural block combined techniques are widely preferred anesthesia. However, the position during the application of these techniques causes severe pain. IV opioid administration or peripheral blocks may be applied to the patient to prevent pain caused by the position. The fascia iliaca compartment block and the femoral nerve block are two of the peripheral nerve blocks used to relieve the patient's position pain due to the femur fracture.

The purpose of this study is to compare the effectiveness of this two blocks for relifing positional pain in patient with femoral neck fractures.

\section{METERIAL AND METOD: 60 ASA} physical status I-III patients aged minimum 60 years inculuded in this study. Fascia iliaca compartment block (FICB) performed with USG in Group-1, USG-assisted femoral nerve blockade in group-2. Sensory block times, before and during position VAS (visiual analog score 0-10) scores, patient satisfaction and additional sedation recorded. Satisfaction was asked after the position was given. One patient exculeded from study because of block failure.

After the application, sensory block recorded in every 5 minutes in the first 30 minutes. Sensory block assessing sensory 3-point scale was used. (0: no block, 1: analgesia (no temperature sense, touch sensation positive) 2 : full sensory block (no touch sensation) ) VAS 0-4 and sense scale 1-2 were considered eligible for patient positioning and the time was recorded.

$0.03 \mathrm{mg} / \mathrm{kg}$ midazolam, $1 \mu \mathrm{g} / \mathrm{kg}$ fentanyl IV was administered and recorded for rescue sedation.
RESULTS: In our study, we found that both blocks were effective in reducing the VAS score .

Although no statistically significant difference was found between two groups, in the FNB group, satisfaction level of the patients was higher than the other group. (FNB28; FICB23) In the FCIB group, where there is more of a partial block, the use of sedation is also more.

Comparing VAS to Groups [(Avr.tSS)/Median (Min-Max)]

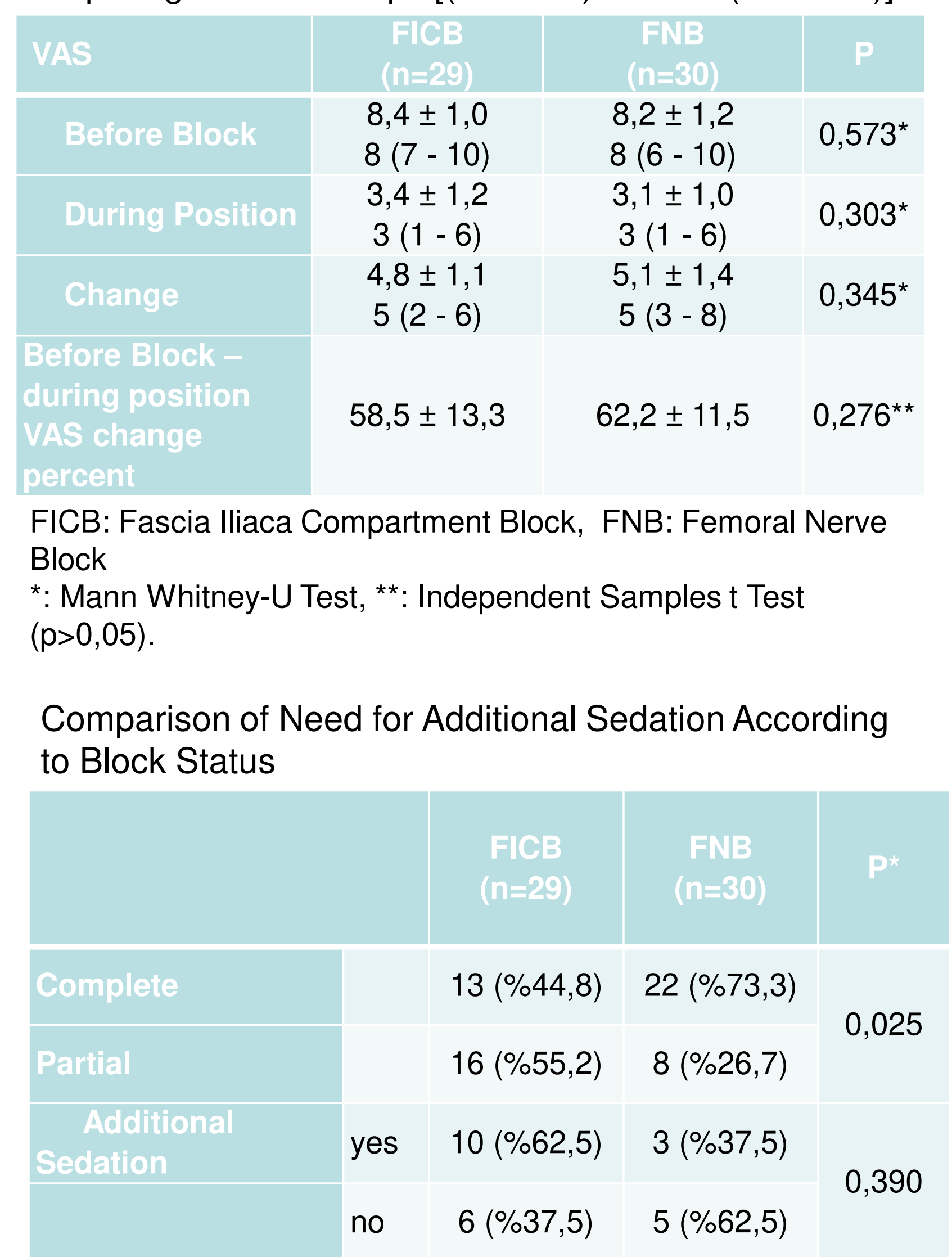

FICB: Fascia Iliaca Compartment Block, FNB: Femoral Nerve Block *: Chi-Square Test $(\mathrm{p}>0,05)$.

CONCLUSION: We conclude that FICB can be used to reduce position pain such as FNB, and patients may be satisfied with these two technics. 